\title{
РЕГИСТР ПАЦИЕНТОВ С ФИБРИЛЛЯЦИЕЙ ПРЕДСЕРДИЙ В КУРСКОЙ ОБЛАСТИ (РЕКУР-ФП): ДИЗАЙН И СТРАТЕГИЯ ИССЛЕДОВАНИЯ
}

\author{
(С Польшакова И.Л., Поветкин С.В.
}

\author{
Кафедра клинической фармакологии Курского государственного медицинского университета, Курск \\ E-mail: potolowa@mail.ru
}

\begin{abstract}
Фибрилляция предсердий (ФП), чаще всего являясь осложнением кардиоваскулярных заболеваний, ухудшает прогноз, увеличивая риск тромбоэмболий (ТЭ), в первую очередь мозгового инсульта. В общей популяции ФП встречается у 1-2\% населения. Высокая распространенность и медико-социальная значимость, с одной стороны, и наличие немногочисленных эпидемиологических данных о клинической характеристике ФП, адекватности и эффективности проводимой терапии, с другой стороны, обусловливают необходимость реализации эффективных способов контроля качества медицинской помощи. Технологической базой этого направления могут служить регистры пациентов с ФП. Для изучения вышеуказанных аспектов проблемы в Курской области инициирован регистр больных с ФП (РЕКУРФП). Исследование позволит получить данные клинического профиля исследуемых пациентов на региональном уровне, определить мероприятия по оптимизации фармакотерапии, а также разработать подходы по снижению развития риска ТЭ и улучшению прогноза у больных с ФП.
\end{abstract}

Ключевые слова: регистр, фибрилляция предсердий, дизайн исследования.

\section{THE REGISTER OF PATIENTS WITH ATRIAL FIBRILLATION IN KURSK REGION (RECUR-AF): DESIGN AND RESEARCH STRATEGY Polshakova I.L., Povetkin S.V.}

Department of Clinical Pharmacology of Kursk State Medical University, Kursk

Atrial fibrillation (AF), a common complication of cardiovascular disease, worsens the prognosis, increasing the risk of thromboembolism (TE), primarily a cerebral stroke. High prevalence and medico-social importance, on the one hand, and the presence of a few epidemiological data on the clinical characteristics of AF, the adequacy and effectiveness of therapy, on the other hand, necessitate the implementation of effective ways of monitoring the quality of care. The registers of patients with AF can serve as a technological base of this direction. To study the above aspects of the problem in Kursk region, the register of patients with AF (RECUR-AF) was initiated. The study will make it possible to obtain data from the clinical profile of the patients being studied at the regional level, to determine the measures for optimizing pharmacotherapy, and to develop approaches to reduce the development of the risk of TE and improve the prognosis in patients with AF.

Keywords: register, atrial fibrillation, study design.

Сердечно-сосудистые заболевания, по данным Всемирной организации здравоохранения, являются одной из ведущих причин смертности среди населения развитых стран мира, в том числе и в России - 635,3 человека на 100 тыс. населения за 2015 г. Около 80\% летальных исходов в группе заболеваний системы кровообращения приходится на долю ишемической болезни сердца, артериальной гипертонии и цереброваскулярных болезней, включая острое нарушение мозгового кровообращения $[7,10]$. Каждый пятый инсульт обусловлен наличием фибрилляции предсердий (ФП) - наиболее распространенного нарушения ритма сердца. В общей популяции ФП встречается у 1-2\% населения и этот показатель, вероятно, возрастет в ближайшие 50 лет. Распространенность фибрилляции предсердий увеличивается с возрастом - от $<0,5 \%$ в возрасте $40-50$ лет до 5-15\% в возрасте 80 лет. Риск развития данной патологии на протяжении жизни составляет около $25 \%$ в возрасте старше 40 лет. В большинстве случаев, являясь осложнением сер- дечно-сосудистых заболеваний, фибрилляции предсердий ухудшает прогноз, значительно увеличивая риск мозгового инсульта, который у больных с ФП часто заканчивается смертью и по сравнению с инсультом другой природы чаще рецидивирует и приводит к более выраженной инвалидизации. Риск смерти у больных инсультом, связанным с ФП, в 2 раза выше, а затраты на лечение возрастают в 1,5 раза $[1,8,11,12]$.

Высокая распространенность и медикосоциальная значимость, с одной стороны, и наличие немногочисленных эпидемиологических данных о клинической характеристике данного вида аритмии, адекватности и эффективности проводимой терапии, с другой стороны, обусловливают необходимость реализации эффективных способов контроля качества медицинской помощи. Технологической базой данного направления могут служить регистры пациентов с ФП.

В России к настоящему времени создан ряд регистров больных с сердечно-сосудистой патологией, включающих пациентов с ФП [2, 3, 4, 
$5,9]$. В большинстве случаев регистры были выполнены на уровне отдельных регионов РФ. Данный факт диктует необходимость широкого внедрения технологии оптимизации медицинской помощи на основе создания регистров в различных субъектах России.

Цель исследования - разработка дизайна и стратегических направлений регистра больных с ФП в Курской области.

\section{МАТЕРИАЛЫ И МЕТОДЫ ИССЛЕДОВАНИЯ}

В основу дизайна регистра больных с ФП были взяты следующие принципы: амбулаторный характер регистра; включение больных, проживающих в г. Курске и в семи районах Курской области; проспективный и динамичный подходы (оценка исходного состояния пациентов при включении в регистр, контроль изучаемых параметров через 6 и 12 месяцев от момента включения в исследование); использование компонентов интегрированного подхода к ведению больных с ФП; автоматизация хранения и анализа данных за счет создания программных продуктов, обеспечивающих функциональность регистра.

Выборка данных для регистра осуществлялась из амбулаторных карт пациентов, проживавших на территории обслуживания медицинских организаций г. Курска и районов Курской области и обращавшихся к терапевтам или кардиологам с сентября 2015 г. по август 2016 г. за медицинской помощью. Критериями включения пациентов в исследование являлся возраст старше 18 лет и наличие любой формы ФП.

Для внесения информации о пациентах изучаемой группы была разработана индивидуальная регистрационная карта, включающая в себя следующие разделы:

- анкетные данные пациента и сведения о медицинской организации, к которой он прикреплен. Персональные данные больных подвергались кодированию и представлены в электронном регистре в обезличенной форме;

- анамнестические сведения, касающиеся основной и сопутствующей патологии;

- клинические данные больного;

- результаты лабораторных исследований;

- результаты инструментальных исследований;

- сведения о назначенных классах лекарственных средств, конкретных препаратах и их суточной дозе.

Учитывая специфику включенных в регистр пациентов, у них проводится расчет риска тромбоэмболических осложнений по шкале $\mathrm{CHA}_{2} \mathrm{DS}_{2} \mathrm{VASc}$, балльная оценка риска кровотечений по шкале HAS-BLED.
Повторная оценка состояния пациентов, проводимая в соответствии с дизайном работы, подразумевала мониторирование частоты и причины госпитализаций; развития инвалидности, полученной с момента включения в регистр; исходов заболевания, а также изменения фармакотерапии.

Вышеуказанные разделы регистрационной карты были трансформированы в электронный вариант базы, помимо которой в регистре был создан блок статистической обработки данных, позволяющий проводить автоматический расчет показателей с учетом используемых фильтров (параметров).

После завершения периода набора пациентов с ФП, в электронную базу были внесены сведения о 896 исследуемых, из них 435 мужчин и 461 женщина, 212 человек из Курской области и 684 человека из г. Курска; средний возраст пациентов в общем по регистру составил $69,1 \pm 10,9$ года, в городе - 69,3 $\pm 11,1$ года, в области $-68,5 \pm 10,3$ года.

\section{РЕЗУЛЬТАТЫ ИССЛЕДОВАНИЯ И ИХ ОБСУЖДЕНИЕ}

Как известно, ФП чаще всего выступает осложнением кардиоваскулярных заболеваний, ухудшая прогноз путем значительного увеличения риска развития тромбоэмболических осложнений, в том числе и инсульта. Повлиять на прогноз представляется возможным с помощью лечения сердечно-сосудистых заболеваний, таких как артериальная гипертензия, сердечная недостаточность, ишемическая болезнь сердца и др., и назначения адекватной антикоагулянтной терапии после оценки риска кровотечений и тромбоэмболий. В созданном регистре особое внимание уделяется улучшению долгосрочного прогноза благодаря использованию антикоагулятной терапии. В большинстве ранее проведенных исследований, включающих больных с ФП, отмечается высокая коморбидность исследуемой когорты пациентов и недостаточное назначение им антикоагулянтов, несмотря на наличие показаний $[2,3,4,5,8]$. Это может быть объяснено рядом факторов, в т. ч. недостаточной информированностью врачей о тактике и стратегии ведения пациентов с ФП в сложных клинических ситуациях. В последних международных и национальных рекомендациях по диагностике и лечению ФП важное значение придается интегрированному подходу к ведению пациентов с указанной патологией (табл. 1) [1, 12]. 
Компоненты интегрированного подхода к лечению больных с ФП

\begin{tabular}{|c|c|c|c|}
\hline Вовлечение пациента & $\begin{array}{c}\text { Междисциплинарные } \\
\text { команды }\end{array}$ & $\begin{array}{c}\text { Технологические } \\
\text { возможности }\end{array}$ & $\begin{array}{c}\text { Контроль всех направлений } \\
\text { лечения }\end{array}$ \\
\hline $\begin{array}{l}\text { •Центральная роль } \\
\text { пациента в процессе } \\
\text { лечения } \\
\text { •Обучение пациентов } \\
\text { •Одобрение и поддержка } \\
\text { самостоятельного } \\
\text { контроля за лечением } \\
\text { •Советы и обучение по } \\
\text { изменению образа } \\
\text { жизни и коррекции } \\
\text { факторов риска } \\
\text { •Совместное принятие } \\
\text { решений }\end{array}$ & $\begin{array}{l}\text { •Совместная работа } \\
\text { врачей общего профиля } \\
\text { (терапевты, } \\
\text { кардиологи), } \\
\text { аритмологов и врачей } \\
\text { смежных } \\
\text { специальностей } \\
\text { •Эффективное } \\
\text { сочетание общения, } \\
\text { обучение и опыта }\end{array}$ & $\begin{array}{l}\text { •Информация о ФП } \\
\text { •Помощь в принятии } \\
\text { решений } \\
\text { •Анкетирование и } \\
\text { навыки общения } \\
\text { •Использование } \\
\text { пациентами и врачами } \\
\text { •Наблюдение за } \\
\text { соблюдением } \\
\text { рекомендаций и } \\
\text { эффективностью } \\
\text { лечения }\end{array}$ & $\begin{array}{l}\text { •Организованная поддержка } \\
\text { в изменении образа жизни } \\
\text { •Антикоагулянтная терапия } \\
\text { •Контроль частоты } \\
\text { сердечных сокращений } \\
\text { •Антиаритмическая терапия } \\
\text { •Катетерные или } \\
\text { хирургические } \\
\text { вмешательства (аблация, } \\
\text { имплантация окклюдера } \\
\text { ушка левого предсердия, } \\
\text { хирургия ФП) }\end{array}$ \\
\hline $\begin{array}{l}\text { Информированный, } \\
\text { заинтересованный, } \\
\text { уверенный пациент }\end{array}$ & $\begin{array}{l}\text { Совместная работа в } \\
\text { группе специалистов по } \\
\text { долгосрочному } \\
\text { лечению ФП }\end{array}$ & $\begin{array}{l}\text { Помощь в принятии } \\
\text { решений в группе } \\
\text { специалистов по ФП }\end{array}$ & $\begin{array}{l}\text { Принятие решений о } \\
\text { комплексном лечении в } \\
\text { группе специалистов по ФП }\end{array}$ \\
\hline
\end{tabular}

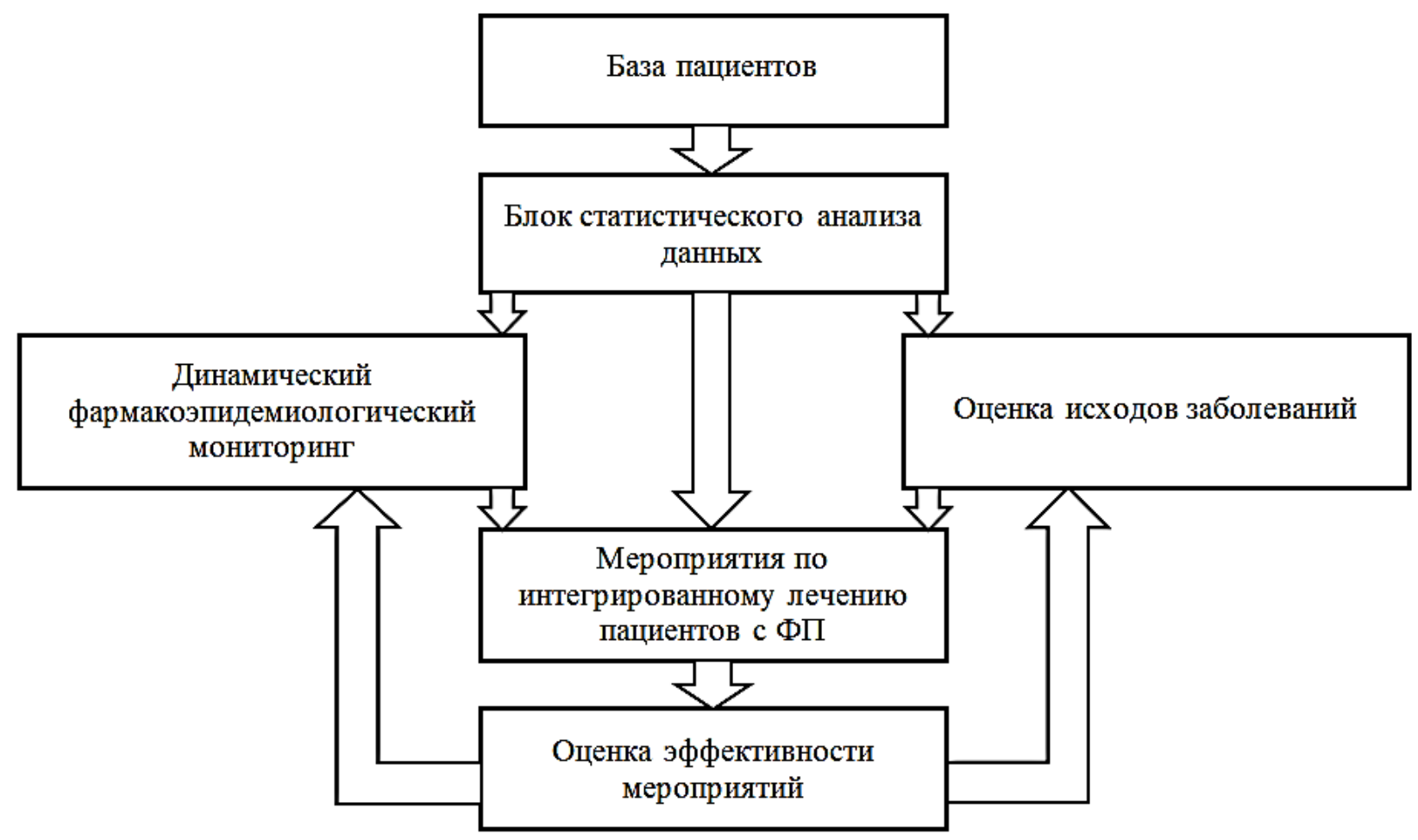

Рис. 1. Дизайн регистра РЕКУР-ФП.

Одним из направлений реализации интегрированного подхода является использование технических средств поддержки фармакотерапевтического процесса. Например, программное обеспечение, облегчающее принятие клинических решений, может способствовать внедрению принципов доказательной медицины и улучшить результаты лечения. С учетом вышеизложенного, наряду с формированием регионального регистра больных с ФП, была разработана и передана в практическое использование в медицинские организации Курской области (в виде практического руководства) система поддержки принятия фармакотерапевтических решений по антитромботической терапии пациентов с ФП в различных клинических ситуациях [6]. Кроме того, был создан и зарегистрирован электронный вариант указанных практических рекомендаций в виде приложения к мобильным устройствам. Данные мероприятия являлись составной частью общей 
концепции построения регистра больных с ФП Курской области (рис. 1).

Таким образом, учитывая поставленные задачи, принципы формирования и функционирования созданного регистра пациентов с ФП, будут получены данные, характеризующие состояние фармакотерапевтического процесса изучаемой патологии в Курском регионе; оценены исходы заболевания; проведено мониторирование динамики исследуемых параметров, в т.ч. в процессе реализации компонентов интегрированного подхода к ведению пациентов с ФП.

\section{ЛИТЕРАТУРА}

1. Диагностика и лечение фибрилляции предсердий. Рекомендации РКО, ВНОА и АССХ (2017) [Электронный ресурс] // WEB-медицина. - Режим доступа:

https:/vnoa.ru/upload/edition_june2017/4_fp.pdf, свободный (27.10.2017).

2. Кореннова О.Ю., Мальцев С.Н., Петренко А.В., Булахова Е.Ю. Фибрилляция предсердий в реальной клинической практике: уроки одного регионального регистра // Трудный пациент. - 2015. T. 13, № 4. - C. 8-11.

3. Лукьянов М.М., Бойцуов С.А., Якушин С.С., Мариевич С.Ю., Воробьев А.Н., Загребельный А.В., Харлап М.С., Переверзева К.Г., Правкина Е.А., Сердюк С.Е., Деев А.Д., Кудряшов Е.Н. Диагностика, лечение, сочетанная сердечно-сосудистая патология и сопутствующие заболевания у больных с диагнозом «фибрилляция предсердий» в условиях реальной амбулаторно-поликлинической практики (по данным Регистра КардиоВАскулярных Заболеваний РЕКВАЗА) // Рациональная фармакотерапия в кардиологии. - 2014. - Т. 10, № 4. С. 366-378.

4. Мариевич С.Ю., Кутишенко Н.П., Суворов А.Ю., Гинзбург М.Л., Деев А.Д., Бойцов С.А. Характеристика пациентов с мозговым инсультом или транзиторной ишемической атакой, включенных в регистр ЛИС-2 // Рациональная фармакотерапия в кардиологии. - 2015. - № 11. - С. 18-24.

5. Мариевич С.Ю., Навасардян А.Р., Кутишенко Н.П., Дроздова Л.Ю., Захарова А.В., Киселева Н.B. Опыт изучения фибрилляции предсердий на базе регистра ПРОФИЛЬ // Кардиоваскулярная терапия и профилактика. - 2014. - Т. 13, № 2. C. 35-39.
6. Поветкин С.В., Польшакова И.Л. Система поддержки принятия фармакотерапевтических решений по антитромботической терапии больных с фибрилляцией предсердий в различных клинических ситуациях. Практическое руководство для врачей, ординаторов, студентов медицинских вузов. - Курск : КГМУ, 2017. - 29 с.

7. Российский статистический ежегодник. 2016: Стат. сб. / под ред. А.Е. Суринова - М. : Росстат, 2016. $725 \mathrm{c}$.

8. Сулимов В.А., Голицыьн С.П., Панченко Е.П., Попов С.В., Ревишвили А.Ш., Шубик Ю.В., Явелов И.С. Диагностика и лечение фибрилляции предсердий. Рекомендации РКО, ВНОА и АССХ (2012) // Российский кардиологический журнал. 2013. - № 4 - С. 1-100.

9. Терещенко С.Н., Жиров И.В., Романова Н.В., Осмоловская Ю.Ф., Голицин С.П. Первый российский регистр больных хронической сердечной недостаточностью и фибрилляцией предсердий (РИФ-ХСН): дизайн исследования // Рациональная фармакотерапия в кардиологии. - 2015. - № 11. C. 577-581.

10. Чазова И.Е., Ощелкова Е.В. Опыт борьбы с сердечно-сосудистыми заболеваниями в России // Аналитический вестник. - 2015. - № 44 - С. 4-8.

11. January C.T., Wann L.S., Alpert J.S., Calkins H., Cigarroa J.E., ClevelandJ.C., Conti J.B., Ellinor P.T., Ezekowitz M.D., Field M.E., Murray K.T., Sacco R.L., Stevenson W.G., Tchou P.J., Tracy C.M., Yancy C.W. 2014 AHA/ACC/HRS Guideline for the Management of Patients With Atrial Fibrillation: Executive Summary // Circulation. - 2014. Vol. 130, N 23. - P. 2071-2104. - doi: 10.1161/CIR.0000000000000040.

12. Kirchhof P., Benussi S., Kotecha D., Ahlsson A., Atar D., Casadei B., Castella M., Diener H.C., Heidbuchel H., Hendriks J., Hindricks G., Manolis A.S., Oldgren J., Popescu B.A., Schotten U., Van Putte B., Vardas P., Agewall S., Camm J., Baron Esquivias G., Budts W., Carerj S., Casselman F., Coca A., De Caterina R., Deftereos S., Dobrev D., Ferro J.M., Filippatos G., Fitzsimons D., Gorenek B., Guenoun M., Hohnloser S.H., Kolh P., Lip G.Y., Manolis A., McMurray J., Ponikowski P., Rosenhek R., Ruschitzka F., Savelieva I., Sharma S., Suwalski P., Tamargo J.L., Taylor C.J., Van Gelder I.C., Voors A.A., Windecker S., Zamorano J.L., Zeppenfeld K. 2016 ESC Guidelines for the management of atrial fibrillation developed in collaboration with EACTS // Eur Heart J. - 2016. - Vol. 37, N 38. P. 2893-2962. - doi:10.1093/eurheartj/ehw210. 